\title{
Factores que afectan el desempeño financiero de las empresas del sector de las confecciones textiles de Cúcuta.
}

Gerson Rueda Vera ${ }^{1}$

Recibido:

Octubre 10 de 2009

Aceptado:

Mayo 10 de 2010
${ }^{1}$ Profesor adscrito al Plan de Estudios de Contaduría Pública, Facultad de Ciencias Empresariales de la Universidad Francisco de Paula Santander. e-mail: gersonrueda@ufps.edu.co

\section{Resumen}

En la presente investigación se planteó como propósito fundamental, determinar la incidencia de la devaluación del Bolívar en la liquidez y la rentabilidad del sector de las confecciones textiles en el municipio de San José de Cúcuta (Norte de Santander - Colombia). Para lo cual se utilizó el tipo de investigación descriptiva correlacional, teniendo como población 50 empresas del área metropolitana, registradas legalmente en la Cámara de Comercio, donde se tomó la totalidad de empresas que conformaron la población para la muestra; se diseño un instrumento en forma de cuestionario contentivo de 25 preguntas, además parte de la investigación fue documental; se destaca que la devaluación del Bolivar conlleva a una situación de iliquidez y baja rentabilidad para las empresas del sector, adicionalmente las empresas no cuenta con financiación y además no tienen una planeación para mitigar este efecto en sus finanzas, por ello se recomienda realizar un programa de financiamiento donde se agrupen; solicitar asesorías tecnológicas y buscar mayor automatización, para reducir costos y ser más competitivos.

Palabras Clave: Devaluación, liquidez, rentabilidad, dólar peso bolívar, confecciones textiles.

\section{Abstract}

This research was the fundamental purpose, to determine the effect of the devaluation of the Bolivar on liquidity and profitability of the textile apparel sector in the municipality of San José de Cúcuta (Norte de Santander - Colombia). For which type of research used descriptive correlational as population having 50 companies in the metropolitan area, legally registered at the Chamber of Commerce, where he took all the companies that made up the population for the sample was designed as an instrument a questionnaire which contains 25 questions, also part of the investigation was documentary stresses that the devaluation of the Bolivar lead to a situation of illiquidity and low profitability 
of the sector stop the company additionally has no business funding and have no further planning to mitigate this effect on your finances, it is recommended to make a funding program which are grouped together, seek advice and seek increased automation technology to reduce costs and become more competitive.

Key Words: Devaluation, liquidity, profitability, dollar peso bolivar, clothing.

\section{Introducción}

E En la dinámica empresarial de la actualidad, los lideres viven diariamente situaciones en las cuales la planeación financiera es una salida a dichas instancias, recordando que la organización tiene procesos administrativos los cuales permiten ubicarla en un futuro cercano, por tanto es necesario tener claro los objetivos para conocer cómo llegar a ellos, en donde la dirección de la empresa encaminará toda la organización buscando soluciones a la situación presentada por la incertidumbre y las medidas tomadas por la política monetaria internacional frente a la política monetaria Colombiana.

En el caso de Venezuela, el decreto 2304 de 2003, establece un sistema de control cambiario con tasa fija, cuya misión es administrar las divisas y todo lo concerniente con el comercio internacional (Banco de la República, 2007) y es normal que la devaluación del bolívar frente al peso traiga consigo crisis, pues al percibir menores ingresos impactan la rentabilidad y la liquidez de las industrias de las confecciones. 14 De acuerdo a lo expuesto por Arenas (2006), el sector industrial debe acogerse al Plan Estratégico Exportador Regional liderado por PROEXPORT y el Comité Asesor de Comercio Exterior (CARCE), que orienta el aumento de ofertas exportables, niveles de productividad y competitividad, permitiendo identificar las verdaderas oportunidades que tienen los productos de la industria local frente a otros mercados, además Ramírez (2002), concluye que el sector de las confecciones es una fortaleza para la industria manufacturera de la ciudad, porque han logrado niveles de organización y desarrollo en cuanto a la actividad, y se ve reflejado en el reconocimiento de la calidad de sus productos.

Así mismo Duque (2005), establece que en el estado Táchira (Venezuela) proliferan las microempresas del sector confecciones, con características más de economía informal que formal, debido a que algunos empresarios Colombianos que han cruzado la frontera buscan protegerse de la devaluación del bolívar.

\section{Referentes teóricos}

Uno de los tantos problemas que afronta la economía mundial es la falta de empresas eficientes y con capacidad competitiva, por las divisas recibidas como exportadores. De acuerdo a Levi (1997), las divisas existen porque los países utilizan distintas unidades monetarias legales dentro de su territorio, careciendo de valor en otros países, teniendo que realizar una conversión y sobre este aspecto Kozikowski (2000), comenta que después del año 1973 se esforzaron por crear un sistema monetario internacional homogéneo, pero en la actualidad no existe tal sistema, ya que los países siguen reglas distintas, respecto de este tema. Madura (2001), afirma que el tipo de cambio variará con el transcurso del tiempo a medida que cambien las curvas de oferta y demanda, sujetas a factores como la tasa de inflación relativa, tasa de interés relativa y nivel de ingreso relativo, en el caso de nuestra moneda la inflación y las tasa de 
interés han tendido a ser constantes, razón por la cual el cambio varia poco y siempre en fortalecimiento del Peso. Estupiñán (2003), señala que la liquidez mide la capacidad que tiene la empresa para pagar sus obligaciones en corto plazo, es decir si no se obtiene suficiente dinero por ventas, debido a que la tasa cambiaria frente al Bolívar este baja, se entraría en iliquidez por parte de las empresas del sector; por otra parte, García (2003), plantea que la rentabilidad que el propietario obtiene corresponde al retorno del capital invertido en la empresa, por tal razón si los ingresos disminuyen se refleja en la utilidad, situación que haría menos rentables a las Mypimes en estudio.

\section{Referentes \\ metodológicos}

La investigación se consideró, según Méndez (2001), como una investigación descriptiva; que se apoyó en una investigación de campo, por cuanto se tomaron datos directamente de los sujetos investigados como son las personas involucradas en las empresas del sector de las confecciones textiles para el periódo 2002-2006. De acuerdo a lo expuesto por: Arias (2004), la variable dependiente es la liquidez y la rentabilidad, dejando como variable independiente la devaluación del Bolívar. La población según Tamayo (1998), está determinada por sus características definitorias y está compuesta por las empresas del sector de las confecciones textiles, que laboran legalmente, y están registradas en la Cámara de Comercio del municipio de San José de Cúcuta para este periódo (20022006), asesoradas por la comisión regional de comercio exterior (CARCE), que contenga un rango de 1 a 80 trabajadores.

El tipo de muestra según Namakforoosh (2006), será seleccionada bajo estricto juicio personal del investigador, siendo la muestra el total de la población, es decir 50 empresas, seleccionadas con un criterio basado en el enfoque de la caracterización y la intencionalidad de la muestra. El instrumento utilizado fue el cuestionario, y se aplicó a los gerentes, administradores o encargados de las empresas seleccionadas, donde los ítems están relacionados con los objetivos específicos de la investigación, y se validó de acuerdo con Hernández, Fernández y Baptista (1998). La validez en términos generales, se refiere al grado en que un instrumento realmente mide la variable que pretende medir; en la encuesta se determinó por el juicio de tres (3) expertos. Para el proceso de análisis y presentación de los datos, se utilizarón hojas de cálculo y el software estadístico SPSS versión 10.0.

\section{Resultados y discusión}

Tabla 1. Relación monetaria Bolívar - Dólar - Peso.

\begin{tabular}{|l|c|c|c|c|c|}
\hline $\begin{array}{l}\text { AÑo } \\
\text { Conceptos }\end{array}$ & $\mathbf{2 0 0 2}$ & $\mathbf{2 0 0 3}$ & $\mathbf{2 0 0 4}$ & $\mathbf{2 0 0 5}$ & $\mathbf{2 0 0 6}$ \\
\hline US - Bolívares & 1400 & 1600 & 1920 & 2150 & 2.150 \\
\hline US - Pesos & 2505 & 2878 & 2628 & 2321 & 2358 \\
\hline Bolívares - Pesos & 2,10 & 1,21 & 0,88 & 0,83 & 0,82 \\
\hline
\end{tabular}

Fuente: datos ordenados por: RUEDA (2009). Banco de la República (2008)

En base a los datos observados en la tabla 1, se observa la fluctuación monetaria, en donde existe una variación de la moneda, en la relación: dólar - bolívar, dólar - peso y bolívar - peso, teniendo un comportamiento correlacional, donde la moneda independiente es el dólar y las monedas dependientes son: el bolívar, que su variación frente al dólar aumento la pérdida de valor monetaria en un $53,57 \%$ durante el periodo analizado; por otra parte está el peso, que sostuvo una recuperación del valor monetario frente al dólar en un 5,87\% y en la relación bolívar - peso se reflejan dichas variaciones en el fortalecimiento de $60,95 \%$ del valor monetario del peso frente al valor del bolívar, lo que demuestra que las monedas analizadas están relacionadas en sus variaciones.

Al procesar la información suministrada 
Factores que afectan el desempeño financiero de las empresas del sector de las confecciones textiles de Cúcuta

por las diferentes empresas se encontró que el $88 \%$ de estas tienen entre 1 y 25 trabajadores, es decir que son pequeñas en su mayoría; además el $74 \%$ opina que la fabricación de sus productos es de buena calidad, el 48\% opinó que no tiene financiamiento a bajos intereses por parte del gobierno, el 36\% tienen problemas para obtener sus materias primas, debido a que en su mayoría están al interior del país, el 54\% de los encuestados cree que su mayor aporte a la sociedad son los impuestos, en promedio el 74\% de los obreros pertenecientes a las empresa encuestadas tienen capacitación técnica de los procesos productivos.

Tabla 2. Comportamiento de la líquidez de las empresas.

\begin{tabular}{|l|c|c|c|c|c|}
\hline & Año 2002 & Año 2003 & Año 2004 & Año 2005 & Año 2006 \\
\hline Buena & $25 \%$ & $34 \%$ & $46 \%$ & $62 \%$ & $60 \%$ \\
\hline Regular & $48 \%$ & $44 \%$ & $50 \%$ & $30 \%$ & $32 \%$ \\
\hline Mala & $27 \%$ & $22 \%$ & $4 \%$ & $8 \%$ & $8 \%$ \\
\hline Total & $100 \%$ & $100 \%$ & $100 \%$ & $100 \%$ & $100 \%$ \\
\hline
\end{tabular}

Según se observa en los datos ordenados en la tabla 2, el comportamiento de la líquidez en las empresas del sector ha sido fluctuante, sin embargo, en la opinión de los encuestados, a partir del año 2005 se da un incremento de la líquidez, esto como consecuencia de una serie de medidas económicos aplicadas como vía de exportación hacia la República Bolivariana de Venezuela, a través de la comisión administrativa de divisas (CADIVI), esto dio como resultado una tasa de cambio oficial más alta, comparada con la ofrecida por Asocambios, lo cual incentivo las ventas.

Gráfico 1. Factores que afectan el capital de trabajo en las empresas del sector.

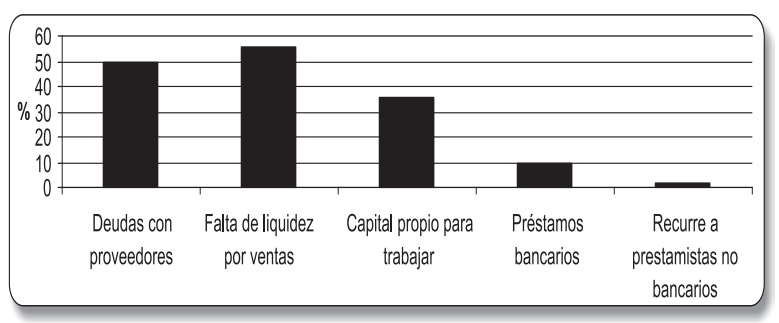

Del gráfico 1, se observa que el 55\% de los encuestados opinaron falta de líquidez por la disminución en las ventas, lo cual es debido a la desaceleración de la economía afectando su capital de trabajo, esta disminución en las ventas es porque uno de los mercados más grandes para sus productos es Venezuela y al bajar la tasa de cambio del bolívar hay menos compradores, adicionalmente las deudas con proveedores afectan el capital de trabajo de las empresas, esto quiere decir que debe cubrir deudas a corto plazo y las empresas no han generado el efectivo necesario para responder; donde lo anterior guarda coherencia con la presentación del grafico 1 .

Gráfico 2. Aspectos financieros que preocupan a las empresas del sector.

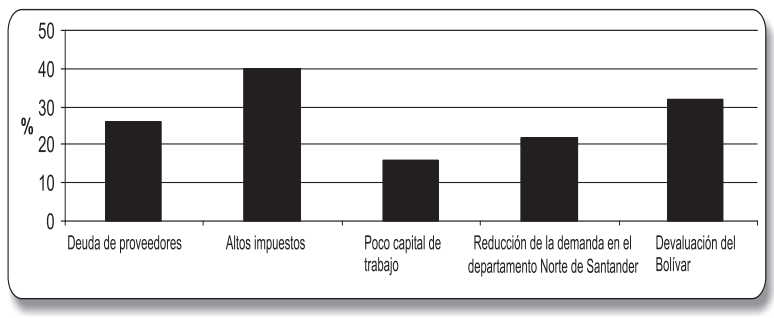

Entre los aspectos financieros que más preocupan a los empresarios del sector confecciones textiles (ver gráfico 2) están las altas tasas impositivas decretadas por el gobierno nacional con 40\%; también es de importancia la devaluación del bolívar, con un $32 \%$ debido a que se abaratan los productos que ingresan ilegalmente de Venezuela y no se pueden sostener los ingresos, ya que se debe cumplir con gastos alusivos a los impuestos, lo que deja en desventaja a los productores nacionales frente a los productos de contrabando, adicionalmente las deudas con proveedores tienen el 26\%; componente que afecta en forma directa la líquidez, puesto que son pasivos a corto plazo que hay que cumplir y representan salidas de dinero; el análisis anterior da respuesta a la pregunta: Los aspectos financieros que preocupan a las empresas del sector, la que se refleja en el grafico 2 . 
Factores que afectan el desempeño financiero de las empresas del sector de las confecciones textiles de Cúcuta

Gráfico 3. Factores que han influido negativamente en el sector confecciones textiles.

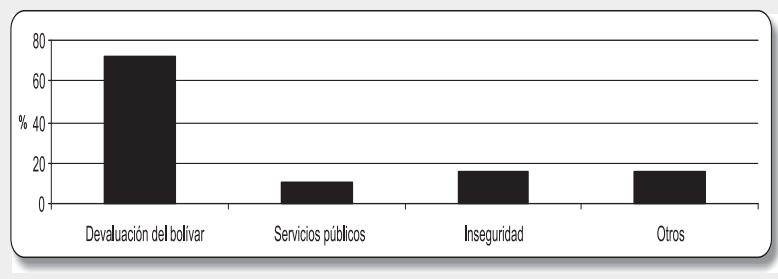

Según los datos ordenados en el grafico 3, en la opinión del $72 \%$ de los empresarios encuestados, el factor que ha perjudicado a las empresas existentes y ha disminuido la creación de nuevas empresas en el sector de las confecciones textiles, es la devaluación del bolívar, que se originó como consecuencia de las políticas económicas cambiarias implementadas por el gobierno de la República Bolivariana de Venezuela.

Grafico 4. Grado en que las empresas del sector son rentables para invertir.

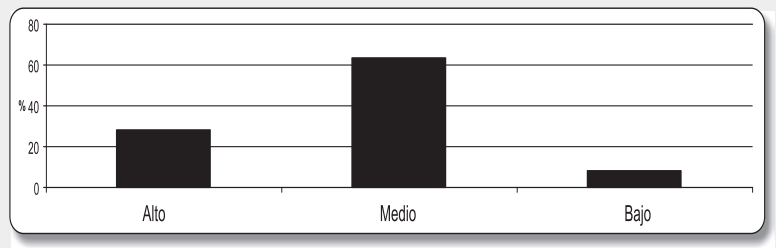

De acuerdo a las respuestas emitidas por los empresarios (ver gráfica 4), más del 60\% de ellos perciben que resulta medianamente rentable invertir en las empresas de confecciones textiles del municipio de Cúcuta, debido a que sus ingresos por ventas decrecen cada vez que se devalúa el bolívar frente al peso, situación que se puede observar claramente en el grafico 4 .

Grafico 5. Planes estratégicos financiero y comercial para afrontar la devolución del bolívar.

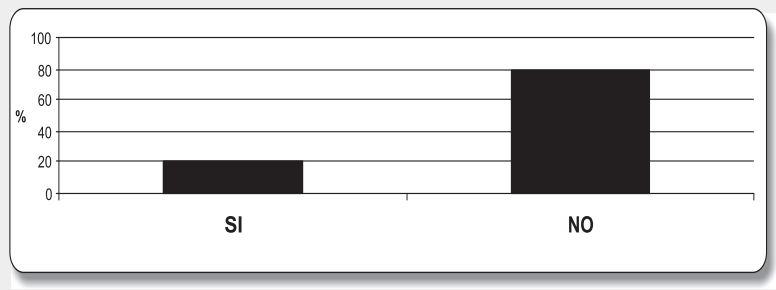

Al observar los resultados reflejados en el grafico 5, se resalta una situación desconcertante ya que en la opinión del $80 \%$ de los empresarios consultados, no disponen de planes estratégicos tanto financiero o comerciales, para afrontar nuevas devaluaciones del bolívar, lo que quiere decir que se continúa esperando una mejoría en el nivel de ventas provenientes del aumento en el valor del bolívar frente al peso.

\section{Conclusiones}

Se concluye que la relación estrecha de la moneda de nuestro país con el dólar, incide en la relación frente al bolívar, la razón es porque si nuestra moneda se fortalece en su relación dólar-pesos, lo hace también en su relación frente al bolívar, por dicha razón la recuperación que ha obtenido el peso en su valor monetario frente al dólar, ha traído como consecuencia una caída constante de la tasa de cambio del bolívar frente al peso.

Por otra parte se pudo concluir que la mayor parte de empresas son pequeñas o Mypimes, y estas poseen debilidades debido a la falta de financiamiento para su funcionamiento productivo, situación que las ha convertido en vulnerables frente a las variaciones de la moneda; también se obtuvo conocimiento que sus materias primas son adquiridas en otras ciudades de Colombia, lo cual no les permite competir en el mercado ya que se ven obligadas a financiarse en la mayoría de los casos con préstamos a terceros o proveedores, situación que conlleva a una mayor iliquidez, debido a que con este tipo de financiación se tienen un costo más elevado del producto, esto sumado al cumplimiento de las altas tasas impositivas de impuestos, adicionalmente el sector en gran parte depende de las ventas de mostrador y no existe la cantidad de clientes esperados por la baja del bolívar, afectando la estructura financiera de las empresas.

La devaluación de bolívar es un factor que ha influido negativamente en las empresas existentes, esta situación ha generado dificultades en la creación de nuevas empresas, y en la actualidad es un sector que no es muy rentable para los inversionista, y 
el comportamiento de su capital de trabajo es débil puesto que sus activos corrientes no son suficientes contra sus pasivos corrientes, $y$ esta situación tiende a permanecer ya que no se posee medidas o planes para superar la crisis de la devaluación del bolívar frente al peso.

\section{Recomendaciones}

Se recomienda establecer planes de protección para enfrentar la incidencia de la devaluación del bolívar, teniendo en cuenta el tamaño que poseen las empresas, puesto que se podrían asociar sin fusionarse, con el propósito de obtener más fácilmente el financiamiento necesario, y así llegar a ser más competitivos.

Es necesario generar confianza en los proveedores con miras a obtener un mayor tiempo de espera para el cumplimiento de sus obligaciones; por otra parte se puede establecer las materias primas que son comunes en varia empresas y conformar un lote mayor, con el ánimo de comprar de contado y obtener precios más bajos.

Es necesario establecer nuevos mercados donde preferiblemente las ventas sean en forma de exportación, pues con esta figura comercial se podrán disminuir las cargas impositivas y adicionalmente se obtendrá una protección frente a las variaciones que pueda tener la moneda frente al bolívar.

\section{Agradecimientos}

Al Magíster: Arcadio Humberto Urbina Méndez, por su acompañamiento en el desarrollo de la Investigación.
Arenas, H. (2006). Fortalecimiento del cluster de la confección en el municipio de San José de Cúcuta. Cúcuta: Alcaldía de Cúcuta - Cámara de Comercio de San José de Cúcuta.

Arias, F. (2004). El proyecto de investigación: Introducción a la metodología científica. (4a ed.). Caracas: Episteme, c.a.

Banco de la República. [Página web en línea]. Disponible en: http://www.banrep. gov.co/docum/publicados/pdf/san_3.pdf, documentode política monetaria en Veñezuela; Consultado el 15 de Junio de 2007.

Duque de V., M. (2005). Mercadeo a través de las tecnologías de información y comunicación, como un factor de apoyo en la competitividad de las Pymes del sector confecciones de la región. Trabajo de grado de maestría no publicado. Universidad Nacional Experimental del Táchira, San Cristóbal.

Estupiñán, R. y Estupiñán, O. (2003). Análisis financiero y de gestión. (1a ed.) Bogotá: Ecoe ediciones Ltda.

García, O. (2003) Valoración de empresas, gerencia del valor y EVA. (2a ed.) Bogotá: Prensa Moderna Impresores S. A.

Hernández, S.; Fernández, C. y Baptista, P. (1998). Metodología de la investigación. (2a ed.). México: MacGraw-Hill.

Kozikowski, Z. (2000). Finanzas internacionales. (1a ed.). México: MacGrawHill.

Levi, M. (1997). Finanzas internacionales. (3a ed.). México: MacGraw- Hill.

Madura, J. (2001). Administración financiera internacional. (6a ed.). México: International Thomson.

Méndez, C. (2001). Metodología. Diseño y desarrollo del procesos de investigación con énfasis en ciencias empresariales. (3a ed.). Bogotá: MacGraw Hill.

Namakforoosh, M (2006) Metodología de la Investigación (2a ed.). México: Limusa

Ramírez A., D. (2002). Análisis situacional y económico de las micro, pyme y gran empresa de los sectores industriales del calzado y las confecciones frente al Plan de Ordenamiento Territorial Municipal y el Plan Estratégico Exportador Regional en su visión de futuro. Tesis de grado Pregrado no publicada. Universidad Francisco de Paula Santander, Cúcuta.

Tamayo, T. (1998). El proceso de investigación científica. (3a ed.). México: Limusa. 